JOURNAL OF THEORETICAL

AND APPLIED MECHANICS

55, 3, pp. 869-881, Warsaw 2017

DOI: $10.15632 /$ jtam-pl.55.3.869

\title{
A NEW AUTOMATED STRETCHING FINITE ELEMENT METHOD FOR 2D CRACK PROPAGATION
}

\author{
Mohammed Bentahar, Habib Benzaama \\ Ecole Nationale Polytechnique, Mechanical Engineering Department, Laboratory of Applied Biomechanics and \\ Biomaterials, ENPO, Oran, Algeria; e-mail: bentahae@yahoo.fr; habenza@yahoo.fr \\ Mohamed Bentoumi \\ Institute of Optics and Precision Mechanics, Ferhat Abbas University, Setif, Algeria; e-mail: hamoudi_10@yahoo.fr \\ MOHAMEd MOKHTARI \\ Ecole Nationale Polytechnique, Mechanical Engineering Department, Laboratory of Applied Biomechanics and \\ Biomaterials, ENPO, Oran, Algeria; e-mail: mokhtarimohamed44@yahoo.fr
}

\begin{abstract}
This work presents a study of crack propagation with a new 2D finite element method with the stretching of the mesh. This method affects at each propagation step new coordinates of each element node of the mesh. The structure is divided to areas and each area has its own coordinate formulas. A program in FORTRAN allows us to create a parametric mesh, which keeps the same number of nodes and elements during different steps of crack propagation. The nodes are stretched using the criterion of maximum circumferential stress (MCS). The fracture parameters such as stress intensity factors in modes I and II and the orientation angles are calculated by solving the problem by the finite element code ABAQUS.
\end{abstract}

Keywords: 2D crack propagation, FEM, stretching finite element method (SFEM), stress intensity factor (SIF)

\section{Introduction}

Today, crack propagation is a large and very complex problem in the study of life of a structure. It is based on the principles of fracture mechanics, in particular, on calculation of the SIF in various modes.

Many analytical formulas exist to determine the SIF. However, these expressions are often developed for different cases of geometry and the solicitation is simple, see Tada and Irwin (1985). Others authors developed numerical methods for modeling crack propagation (Chan et al., 1970; Bouchard et al., 2003). They proposed a solution by the finite element method to model crack propagation problems in $2 \mathrm{D}$. In the recent years, various approaches have been proposed such as the extended finite element method (XFEM), Möes et al. (1999), and methods of generalized finite elements (GFEM), Babuska and Banerjee (2012). Both methods are based on the partition of the unity method proposed by Babuska and Belenk (1997). In addition, the use of mesh regeneration crack schemes improved the estimate of the increment criteria. Bouchard et al. (2000) developed a crack propagation method using a linking technique that optimized the size and quantity of the elements, but the geometry was quite remeshed in each step.

The same method was used for several elastic and plastic examples. Bouchard et al. (2003), Phongthanapanich and Dechaumphai (2004) developed a method that completely reconstructed the mesh between refinement steps using the adaptive mesh and Delaunay triangulations. 
Meyer et al. (2006) proposed a crack propagation method that combined an adaptive iterative solver, mesh refinement and mesh coarsening techniques as well as optimization of the number of nodes.

In exchange, Askes et al. (2001) discussed linkage strategies based on the relocation of nodes for $r$-adaptive and $h / r$-adaptive analysis of crack propagation.

Another approach is the boundary element method (Aliabadi, 1997; Portela et al., 1991; Yan and Nguyen-Dang, 1995), Galerkin method (Belytschko et al., 1994), meshfree element methods (Belytschko et al., 1994; Yan, 2006; Duflot and Nguyen-Dang, 2004) and the finite element method (FEM) Singh et al. (2012).

Numerical methods have been widely used to calculate fracture parameters, including the mechanics of linear elastic plastic-fracture (Bouchard et al., 2003), mechanical dynamics and breaking (Réthoré et al., 2005), tiredness (Miranda et al., 2003) and the spread of quasi-static crack (Khoei et al., 2008). Azocar et al. (2010) proposed a new method for the simulation of crack propagation in solids (LEFM) (2D). They used the Lepp-Delaunay method based on the refinement of the triangular mesh by an algorithm that allowed both generation of the first mesh and local modification of the current mesh during propagation of the crack.

This method uses a technique of displacement extrapolation to calculate $K_{I}$ and $K_{I I}$, and the maximum circumferential stress criterion to calculate the crack propagation angle. Alshoaibi (2015) used the method of extrapolation of the movement to simulate crack propagation in 2D by the finite element method of a linear elastic plate. He also characterized the singularity of the crack tip, and the stress intensity factors around the crack tip. Zaleha et al. (2007) proposed to assess the displacement extrapolation technique (DET) for predicting the stress intensity factor.

The DET is used when the singular element is present at the tip of the crack. It uses differential movements to adjacent nodes through the crack to calculate the SIF. Cho (2015) proposed mixed modes for stress intensity factors of a 2D inclined crack and evaluated them by Petrov-Galerkin natural element method (PG-NE). Murat (2016) presented a comparative study of the finite element method (FEM) and an analytical method for the problem of a plane layered composite containing an internal perpendicular crack.

\section{Law of fatigue crack propagation (Paris and Erdogan, 1963)}

The simplest and the oldest law to model fatigue crack propagation in the two-dimensional case is the law proposed by Paris and Erdogan (1963). It is based on constant amplitude tests for which the propagation velocities appear as a linear function of the variation in the SIF in a log-log diagram. It takes the form

$$
\frac{d A}{d N}=C(\Delta K)^{m}
$$

where $C$ and $m$ are material properties, $A$ is crack length, $N$ is the number of loading cycles and $\Delta K$ variation of the stress intensity factor. $C$ and $m$ are the material constants. The crack propagation length is kept constant during propagation steps.

\section{Crack propagation criteria}

In order to simulate crack propagation under the linear elastic condition, the crack path direction must be determined. There are several methods used to predict the direction of the crack trajectory such as the maximum normal stress theory (or the maximum circumferential stress theory, Erdogan and Sih (1963)) and the minimum strain energy density theory (Sih, 1974). 


\subsection{Maximum circumferential stress criterion (MCSC)}

This criterion is used for elastic materials. It states that the crack propagates in the direction for which the circumferential stress $\sigma_{\theta \theta}$ is maximum. It is a local approach since the direction of crack growth is directly determined by the local stress field along a small circle of radius $r$ centered at the crack tip.

The kinking angle $\alpha$ of the propagating crack can be determined after calculating the values of the stress intensity factors $K_{I}$ and $K_{I I}$.

$$
\alpha=2 \arctan \left[\frac{1}{4} \frac{K_{I}}{K_{I I}} \pm \frac{1}{4} \sqrt{\left(\frac{K_{I}}{K_{I I}}\right)^{2}+8}\right]
$$

where $K_{I}$ and $K_{I I}$ are, respectively, the stress intensity factors corresponding to mode I and mode II loading.

\section{Criterion of Richard 2D}

$$
K_{V}=\frac{K_{I}}{2}+\frac{1}{2} \sqrt{K_{I}^{2}+5.366 K_{I I}^{2}}=K_{I C}
$$

where $K_{V}$ depends on the stress intensity factors $K_{I}$ and $K_{I I}$. It is noticeable that unstable crack growth occurs if $K_{V}$ exceeds the fracture toughness $K_{I C}$.

This criterion has an excellent approximation of the fracture limit surface of the maximum tangential stress criterion (Erdogan and Sih, 1963). The crack kinking angle $\alpha$ can be determined by

$$
\alpha=\mp\left[140^{\circ} \frac{\left|K_{I I}\right|}{\left|K_{I}\right|+\left|K_{I I}\right|}-70^{\circ}\left(\frac{\left|K_{I I}\right|}{\left|K_{I}\right|+\left|K_{I I}\right|}\right)^{2}\right]
$$

whereby for $K_{I I}>0$ the kinking angle $\alpha<0$ and vice versa while always $K_{I}>0$. There are some more criteria, e.g. criterion of Nuismer (1975) or criterion of Amestoy et al. (1980), which are based on the energy release rate and describes the crack growth for 2D-mixed-mode-loading situations.

\subsection{Field of the stresses in the vicinity of the forehead of the crack}

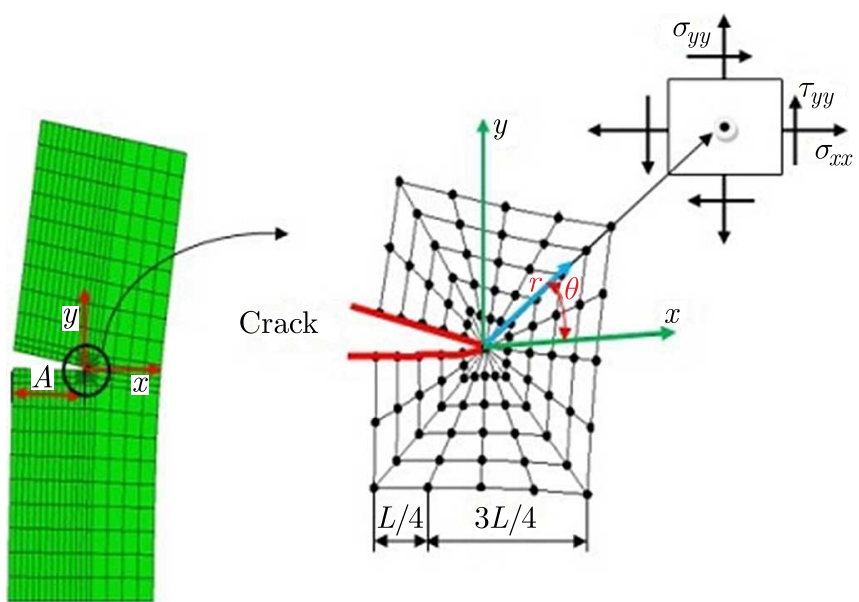

Fig. 1. Field of stresses in the vicinity of the front of the crack 
The general equation of the stress field in 2D near the crack front defined by the stress intensity factor $K$ is given by Tada et al. (2000)

$$
\sigma_{i, j}^{I, I I}(r, \theta)=\frac{K_{I, I I}}{\sqrt{2 \pi r}} f_{i j}(\theta)
$$

$K_{I, I I}$ is the SIF in mode I and II, $\sigma_{i, j}^{I, I I}$ is the associated stress field with mode I.

$K_{I}$ is one that contributes the most to the propagation of the fatigue cracks. Fatigue cracks tend to spread according to the perpendicular direction to the maximum tangential (Erdogan and Sih, 1962)

$$
\begin{aligned}
\sigma_{x x} & =\frac{K_{I}}{\sqrt{2 \pi r}} \cos \frac{\theta}{2}\left(1-\sin \frac{\theta}{2} \sin \frac{3 \theta}{2}\right) \quad \sigma_{y y}=\frac{K_{I}}{\sqrt{2 \pi r}} \cos \frac{\theta}{2}\left(1+\sin \frac{\theta}{2} \sin \frac{3 \theta}{2}\right) \\
\tau_{x y} & =\frac{K_{I}}{\sqrt{2 \pi r}} \sin \frac{\theta}{2} \cos \frac{\theta}{2} \cos \frac{3 \theta}{2}
\end{aligned}
$$

\section{(a)}

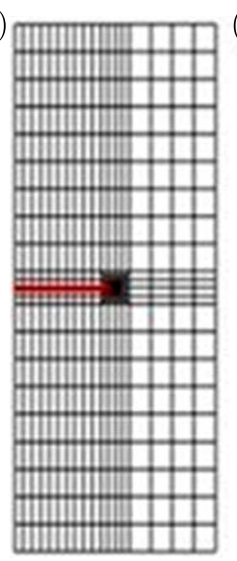

(b)

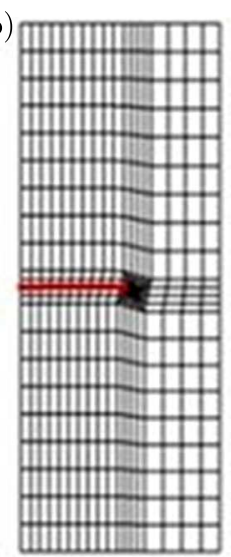

(c)

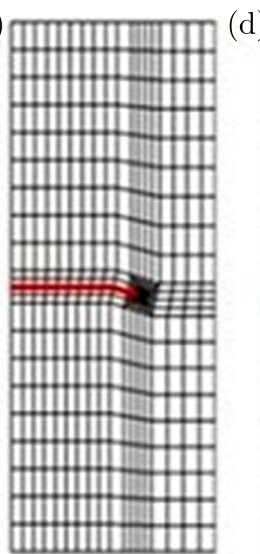

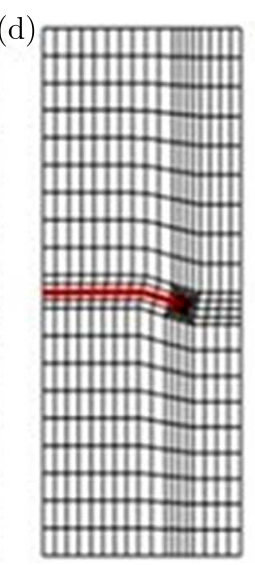

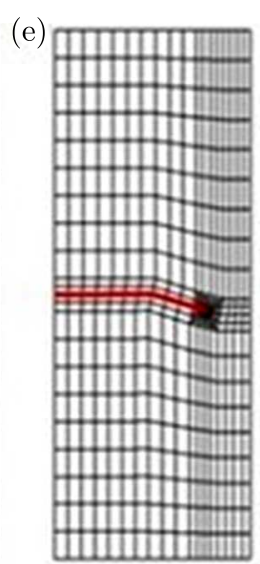

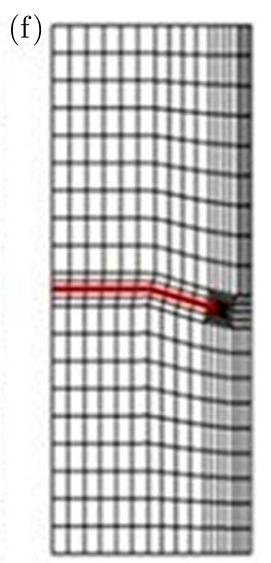

Fig. 2. Stretching finite element method for 5 crack propagation

\section{Illustration of 2D SFEM crack propagation}

The finite element method by the stretching of the mesh (SFEM) generates for each propagation new coordinates to each node through the following steps:

- Creation of singular elements and elements of contours of zone 5, see Fig. 3a by SFEM using the crack propagation law respecting the propagation criterion.

- The elements of zone 1 and 2 are stretched and at each propagation. Column one of these elements moves to the front of the mesh, the zones are divided in two parts, one is zone 1' and the other is area 2', Fig. 3b.

- . The elements of zones 4 and 6 are compressed in each crack propagation, see Fig. 3c.

Point 1 is the center the forehead of the crack and the starting basis for creation of other nodes by this method. The coordinates of this point are given by the following relationship

$$
X(1)=A \quad Y(1)=0
$$

where $A$ is the crack length. 


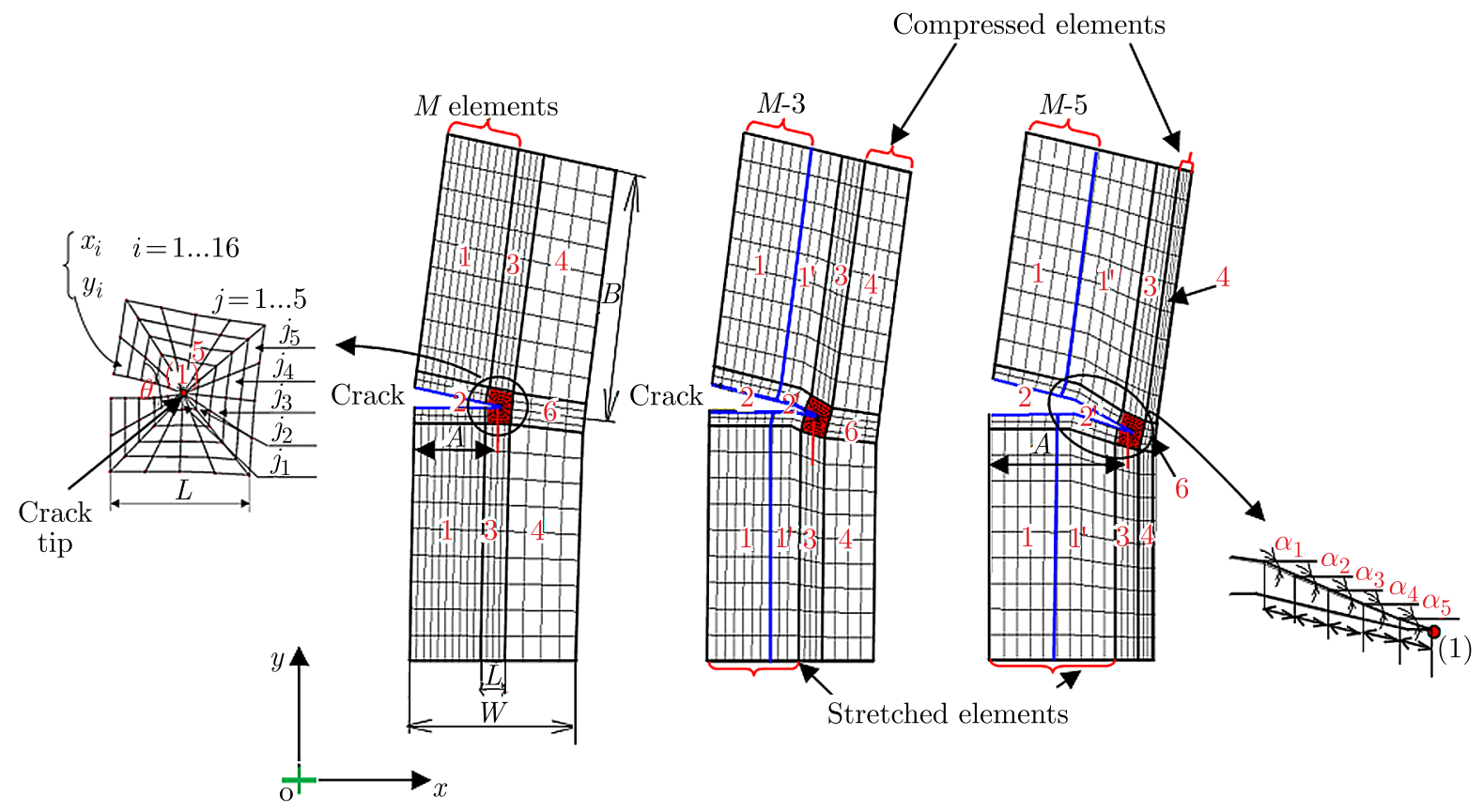

Fig. 3. Schematic illustration of SFEM method: (a) mesh without crack propagation, (b) mesh with 3 crack step propagation, (c) mesh with 5 crack propagations

Singular elements of the CPE4 type are used to determine zone 5. The following equation defines the coordinates of the element nodes in this zone

$$
x_{i}=X(1)-j \frac{L}{t} \cos ((i-1) \theta) \quad y_{i}=Y(1)-j \frac{L}{t} \sin (i \theta)
$$

where $j=1, \ldots, t$ is the number of contour, $i=1, \ldots, 16$ - number of elements, $\theta=\pi / 16-$ angular division.

\section{New coordinates of different areas for $n$ propagations}

Node 1 is the front of the crack; it is taken as the reference for all the other nodes of the mesh. The coordinates of this node for $n$ propagation are given by the following relationship

$$
X(1)=A+\frac{L}{2} \sum_{p=1}^{n}\left(\cos \sum_{k=1}^{p} \alpha_{k}\right) \quad Y(1)=\frac{L}{2} \sum_{p=1}^{n}\left(\sin \sum_{k=1}^{p} \alpha_{k}\right)
$$

where $\alpha$ is the crack orientation angle; $p$ is the number of total crack propagation, $p=1, \ldots, n$; $L$ is length of the crack front.

From the first propagation, area 1 is divided into two areas: 1 and 1', see Fig. 3b. The coordinates in the second area can be given by the following formulas

$$
\begin{aligned}
& x_{i}=\frac{1}{M-p}\left\{X(1)-\left[\sum_{p=1}^{n}\left(\cos \sum_{k=1}^{p} \alpha_{k}\right)+S \sum_{p=1}^{n}\left(\sin \sum_{k=1}^{p} \alpha_{k}\right)\right] \frac{L}{2}\right\}(i-1) \\
& y_{j}=-S \frac{L}{2}+\frac{B-\frac{L}{2}}{N}(j-1)
\end{aligned}
$$

where

$$
\begin{aligned}
& i=1, \ldots, 10 \quad \text { and } \quad M=10 \quad p=1, \ldots, n \\
& j=1, \ldots, 9 \quad \text { and } \quad N=9 \quad k=1, \ldots, p
\end{aligned}
$$


and for zone 1', the node coordinates are

$$
\begin{aligned}
x_{i}= & X(1)-\left[\sum_{p=1}^{n}\left(\cos \sum_{k=1}^{p} \alpha_{k}\right)-S \sum_{p=1}^{n}\left(\sin \sum_{k=1}^{p} \alpha_{k}\right)\right] \frac{L}{2} \\
& +\frac{1}{M-p}\left\{\frac{L}{2} \sum_{p=1}^{n}\left(\cos \sum_{k=1}^{p} \alpha_{k}\right)\right\}(i-1) \\
y_{j} & =Y(1)+\left[-S \sum_{p=1}^{n}\left(\cos \sum_{k=1}^{p} \alpha_{k}\right)-\sum_{p=1}^{n}\left(\sin \sum_{k=1}^{p} \alpha_{k}\right)\right] \frac{L}{2} \\
& +\frac{1}{N}\left\{B-\left[\sum_{p=1}^{n}\left(\cos \sum_{k=1}^{p} \alpha_{k}\right)+\sum_{p=1}^{n}\left(\sin \sum_{k=1}^{p} \alpha_{k}\right)\right] \frac{L}{2}\right\}(j-1)
\end{aligned}
$$

where

$$
\begin{array}{llll}
i=1, \ldots, n & \text { and } & M=4 & p=1, \ldots, 5 \\
j=1, \ldots, 9 & \text { and } & N=9 & k=1, \ldots, 5
\end{array}
$$

and $M$ is the number of vertical elements, $N$ is the number of horizontal elements, $B$ is height of the structure, $S$ is sign of the exchange factor between the two zones, the top and the bottom ( $S=-1$ for the top part area and $S=1$ for the bottom part area).

The new coordinates of the elements of area 3 , for $n$ propagation can be written as

$$
\begin{aligned}
x_{i}= & X(1)-\left[\sum_{p=1}^{n}\left(\cos \sum_{k=1}^{p} \alpha_{k}\right)-S \sum_{p=1}^{n}\left(\sin \sum_{k=1}^{p} \alpha_{k}\right)\right] \frac{L}{2} \\
& +\frac{1}{M-p}\left\{\frac{L}{4} \sum_{p=1}^{n}\left(\cos \sum_{k=1}^{p} \alpha_{k}\right)\right\}(i-1) \\
y_{j} & =Y(1)-\left[S \sum_{p=1}^{n}\left(\cos \sum_{k=1}^{p} \alpha_{k}\right)-S \sum_{p=1}^{n}\left(\sin \sum_{k=1}^{p} \alpha_{k}\right)\right] \frac{L}{2} \\
& -\frac{1}{N}\left\{S B+\left[Y(1)-S \sum_{p=1}^{n}\left(\cos \sum_{k=1}^{p} \alpha_{k}\right)+S \sum_{p=1}^{n}\left(\sin \sum_{k=1}^{p} \alpha_{k}\right)\right] \frac{L}{2}\right\}(j-1)
\end{aligned}
$$

where

$$
\begin{array}{llll}
i=1, \ldots, 4 & \text { and } & M=4 & p=1, \ldots, n \\
j=1, \ldots, 9 & \text { and } & N=9 & k=1, \ldots, p
\end{array}
$$

The elements of area 4 are compressed as shown in Fig. 3b. The definition of coordinates of this area may be given by the following relationship:

$$
\begin{aligned}
x_{i}= & X(1)+\left[\sum_{p=1}^{n}\left(\cos \sum_{k=1}^{p} \alpha_{k}\right)+S \sum_{p=1}^{n}\left(\sin \sum_{k=1}^{p} \alpha_{k}\right)\right] \frac{L}{2} \\
& +\frac{1}{M-p}\left\{W-\left[X(1)-S \sum_{p=1}^{n}\left(\cos \sum_{k=1}^{p} \alpha_{k}\right)+S \sum_{p=1}^{n}\left(\sin \sum_{k=1}^{p} \alpha_{k}\right)\right] \frac{L}{2}\right\}(i-1) \\
y_{j} & =Y(1)+\left[-S \sum_{p=1}^{n}\left(\cos \sum_{k=1}^{p} \alpha_{k}\right)+\sum_{p=1}^{n}\left(\sin \sum_{k=1}^{p} \alpha_{k}\right)\right] \frac{L}{2} \\
& +\frac{1}{N}\left\{B-\left[Y(1)-\left[\sum_{p=1}^{n}\left(\cos \sum_{k=1}^{p} \alpha_{k}\right)-\sum_{p=1}^{n}\left(\sin \sum_{k=1}^{p} \alpha_{k}\right)\right] \frac{L}{2}\right]\right\}(j-1)
\end{aligned}
$$


where

$$
\begin{aligned}
& i=1, \ldots, 4 \quad \text { and } \quad M=4 \quad p=1, \ldots, n \\
& j=1, \ldots, 9 \quad \text { and } \quad N=9 \quad k=1, \ldots, p
\end{aligned}
$$

and $C$ is width of the structure.

Area 2 is an area which contains elements of singularity. During the steps of propagation, zone 2 is divided into two zones 2 and 2', see Fig. 3b. Equations (6.6) and (6.7) show the coordinates of area nodes 2 and 2', respectively

$$
\begin{aligned}
& x_{i}=\frac{1}{M-p}\left\{X(1)-\left[\sum_{p=1}^{n}\left(\cos \sum_{k=1}^{p} \alpha_{k}\right)+S \sum_{p=1}^{n}\left(\sin \sum_{k=1}^{p} \alpha_{k}\right)\right] \frac{L}{2}\right\}(i-1) \\
& y_{j}=-S \frac{L}{4}+\frac{L}{2 N}(j-1)
\end{aligned}
$$

where

$$
\begin{aligned}
& i=1, \ldots, 10 \quad \text { and } \quad M=10 \quad p=1, \ldots, 5 \\
& j=1, \ldots, 4 \quad \text { and } \quad N=4 \quad k=1, \ldots, 5
\end{aligned}
$$

and

$$
\begin{aligned}
x_{i}= & X(1)-\left[\sum_{p=1}^{n}\left(\cos \sum_{k=1}^{p} \alpha_{k}\right)+\sum_{p=1}^{n}\left(\sin \sum_{k=1}^{p} \alpha_{k}\right)\right] \frac{L}{2} \\
& +\frac{1}{M-p}\left\{X(1)-\left[\sum_{p=1}^{n}\left(\cos \sum_{k=1}^{p} \alpha_{k}\right)+\sum_{p=1}^{n}\left(\sin \sum_{k=1}^{p} \alpha_{k}\right)\right] \frac{L}{2}\right\}(i-1) \\
y_{j} & =Y(1)+\left[\sum_{p=1}^{n}\left(\cos \sum_{k=1}^{p} \alpha_{k}\right)-\sum_{p=1}^{n}\left(\sin \sum_{k=1}^{p} \alpha_{k}\right)\right] \frac{L}{2} \\
& +\frac{1}{N}\left\{L \sum_{p=1}^{n}\left(\sin \sum_{k=1}^{p} \alpha_{k}\right)-\frac{L}{2} \sum_{p=1}^{n}\left(\sin \sum_{k=1}^{p} \alpha_{k}\right)\right\}(j-1)
\end{aligned}
$$

where

$$
\begin{array}{llll}
i=1, \ldots, 4 & \text { and } & M=4 & p=1, \ldots, n \\
j=1, \ldots, 4 & \text { and } & N=4 & k=1, \ldots, p
\end{array}
$$

The coordinates of the node elements of area 6 can be written as

$$
\begin{aligned}
x_{i}= & X(1)+\left[\sum_{p=1}^{n}\left(\cos \sum_{k=1}^{p} \alpha_{k}\right)-\sum_{p=1}^{n}\left(\sin \sum_{k=1}^{p} \alpha_{k}\right)\right] \frac{L}{2} \\
& +\frac{1}{M-p}\left\{W-\left[X(1)+\sum_{p=1}^{n}\left(\cos \sum_{k=1}^{p} \alpha_{k}\right)-\sum_{p=1}^{n}\left(\sin \sum_{k=1}^{p} \alpha_{k}\right)\right]\right. \\
& \left.-L \sum_{p=1}^{n}\left(\sin \sum_{k=1}^{p} \alpha_{k}\right)\right\}(i-1) \\
y_{j} & =Y(1)+\left[\sum_{p=1}^{n}\left(-\cos \sum_{k=1}^{p} \alpha_{k}\right)+\sum_{p=1}^{n}\left(\sin \sum_{k=1}^{p} \alpha_{k}\right)\right] \frac{L}{2} \\
& +\frac{1}{N}\left\{B-\left[L \sin \alpha_{k}+Y(1)+\left[\sum_{p=1}^{n}\left(-\cos \sum_{k=1}^{p} \alpha_{k}\right)+\sum_{p=1}^{n}\left(\sin \sum_{k=1}^{p} \alpha_{k}\right)\right] \frac{L}{2}\right]\right\}(j-1)
\end{aligned}
$$

where

$$
\begin{array}{lccc}
i=1, \ldots, n & \text { and } & M=4 & p=1, \ldots, n \\
j=1, \ldots, N & \text { and } & N=4 & k=1, \ldots, p
\end{array}
$$




\section{Interface FORTRAN ABAQUS}

In structure of executing the FORTRAN program in interface with the ABAQUS (FEM) is shown in Fig. 4.

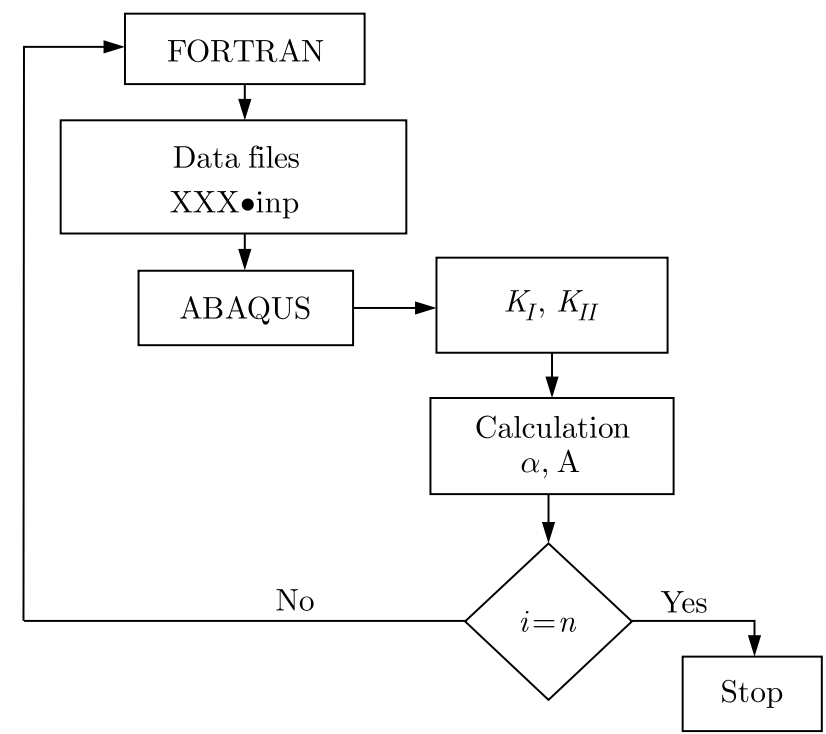

Fig. 4. The structure of the interface between FORTRAN and ABAQUS to calculate $K_{I}, K_{I I}$ and $\alpha$

\section{Numerical model (mesh)}

The structure considered has length $B=8 \mathrm{~mm}$ and width $W=7 \mathrm{~mm}$, the horizontal crack length is $A=3.5 \mathrm{~mm}$, length of the front $L=1 \mathrm{~mm}$. The parametric mesh consists of 478 square CPE4 type elements with four nodes.

The total number of degrees of freedom is equal to 1016. The FORTRAN program for creation of the mesh that will be analyzed by the finite element code ABAQUS has been applied. The steel structure with $E=1 \cdot 10^{7} \mathrm{~Pa}$ and $\nu=0.25$ is subjected to a uniform tensile stress $\sigma=100 \mathrm{MPa}$.

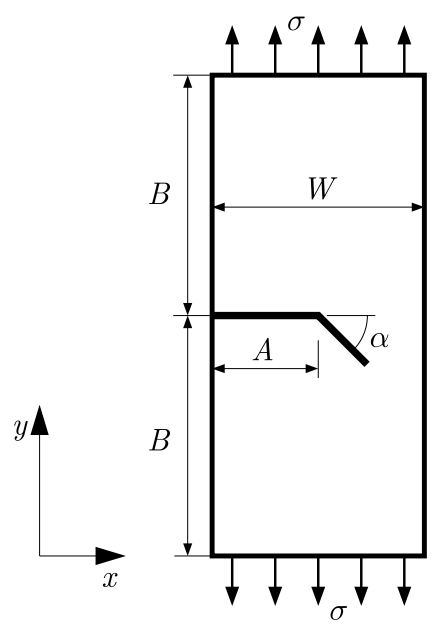

Fig. 5. The specimen with a crack 


\section{Analytical calculation of stress intensity factors}

The analytical stress intensity factor $K_{I}$ for this problem is given (Ewalds and Wanhill, 1989) as

$$
K_{I}=F \sigma \sqrt{A \pi}
$$

where $F$ is the correction factor given by

$$
F=1.12-0.23 \frac{A}{W}+10.6\left(\frac{A}{W}\right)^{2}-21.7\left(\frac{A}{W}\right)^{3}+30.4\left(\frac{A}{W}\right)^{4}
$$

where the stress intensity factor $K_{I I}$ is calculated by the relation

$$
K_{I} \sin \theta+K_{I I}(3 \cos \theta-1)=0
$$

The analytical values of calculated stress intensity factors are compared with the numerical results obtained by SFEM.

Table 1. Comparison of analytical results and SFEM for stress intensity factor $K_{I}$ and $K_{I I}$

\begin{tabular}{|c|c|c|c|c|c|c|}
\hline $\begin{array}{c}\alpha \\
{\left[{ }^{\circ}\right]}\end{array}$ & $\begin{array}{c}K_{I}^{\mathrm{ANAL}} \\
{[\mathrm{MPa} \sqrt{\mathrm{m}}]}\end{array}$ & $\begin{array}{c}K_{I}^{\mathrm{SFEM}} \\
{[\mathrm{MPa} \sqrt{\mathrm{m}}]}\end{array}$ & $\begin{array}{c}\text { Error } \\
{[\%]}\end{array}$ & $\begin{array}{c}K_{I I}^{\mathrm{ANAL}} \\
{[\mathrm{MPa} \sqrt{\mathrm{m}}]}\end{array}$ & $\begin{array}{c}K_{I I}^{\mathrm{SHEM}} \\
{[\mathrm{MPa} \sqrt{\mathrm{m}}]}\end{array}$ & $\begin{array}{c}\text { Error } \\
{[\%]}\end{array}$ \\
\hline \hline-14.76 & 31.58 & 33.03 & -4.591 & 4.232 & 4.427 & -4.608 \\
\hline-0.643 & 42.90 & 42.84 & 0.139 & 0.2408 & 0.2404 & 0.166 \\
\hline 0.8313 & 56.95 & 55.90 & 1.844 & -0.4132 & -0.4056 & 1.839 \\
\hline 1.577 & 75.68 & 76.65 & -1.282 & -1.041 & -1.056 & -1.441 \\
\hline 2.049 & 114.51 & 113.9 & 0.532 & -2.049 & -2.038 & 0.54 \\
\hline
\end{tabular}

\section{Evaluation of the SIFs as functions of area 5}

Zone 5 (singular area) is the most important area, see Fig. 3a. This zone consists of singular elements and other constituent element contours. Its shape is a square of side $L$. This area can be parametrically varied in order to optimize the mesh.

Figures $6 \mathrm{a}$ and $6 \mathrm{~b}$ show the change in crack length $A$ depending on the size $L$ of the front of singularity zone 5 . The stress intensity factors $K_{I}$ and $K_{I I}$ are compared with theoretical values for each stage of propagation of the rift. The interval optimization of zone 5 is in an interval $L$ between $0.1 \leqslant L \leqslant 1.1$. This comparison shows a good correlation between the analytical method proposed by Ewalds and Wanhill (1989) and the method of stretching the (SFEM) mesh for the value of $L=1.0 \mathrm{~mm}$. There is a very good correspondence between the results obtained in the case of $L=1.0 \mathrm{~mm}$ compared to other values of $L$.

The results given by the SFEM are close to those of the analytical solution. The results are obtained for different tilt angles for different crack ratios $A / W$ between 0.5 and 0.8 . Figure $7 \mathrm{a}$, shows the evolutions between the SIF and the crack length $A$, and Fig. $7 \mathrm{~b}$ depicts an increases in this ratio for which the intensity factor $K_{I}$ increases and $K_{I I}$ decreases.

Figure 8 illustrates the variation of the angle of inclination $\alpha$ estimated at each increment of length of the crack. This comparison shows a good comparison between the two criteria, the criterion of Richard and the MCS criterion we used in our study.

For different values of SIF for the two numerical models and the analytical one, Fig. 7b shows similar values for $K_{I}$ and $K_{I I}$ as functions of the crack length $A$. Similarly, see Fig. 7a for the ratio $A / W$ one can observe that there is an increase in length and crack orientation angle, which 

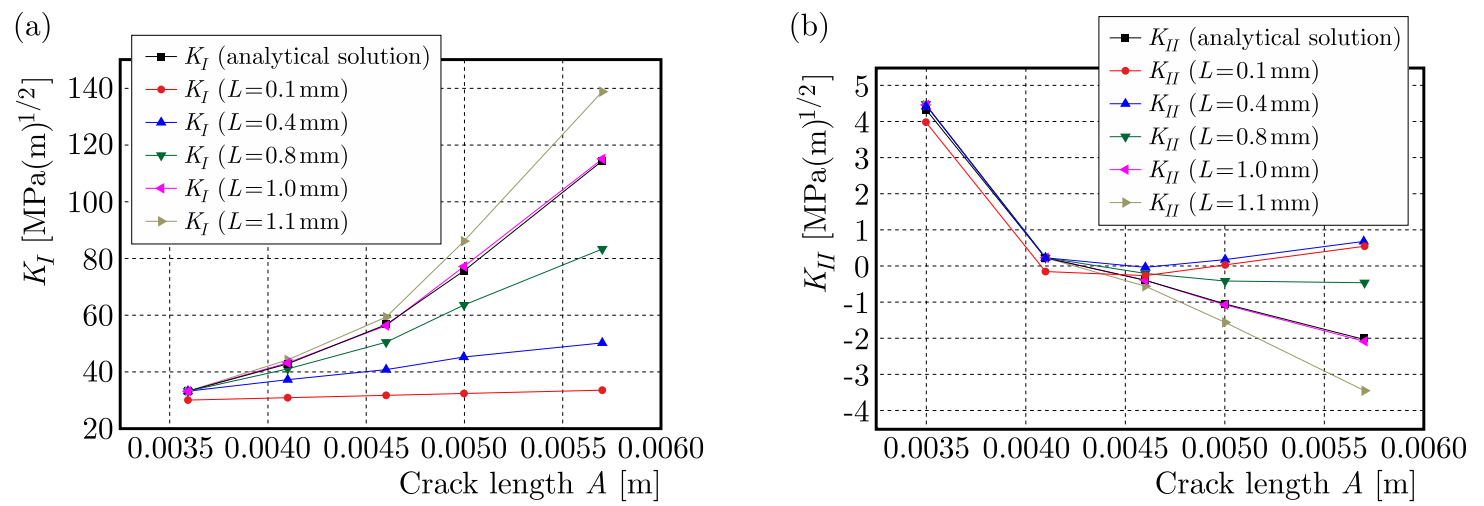

Fig. 6. Variation of SIF along crack length $A$ for different values of $L$ : (a) $K_{I}$, (b) $K_{I I}$

(a)

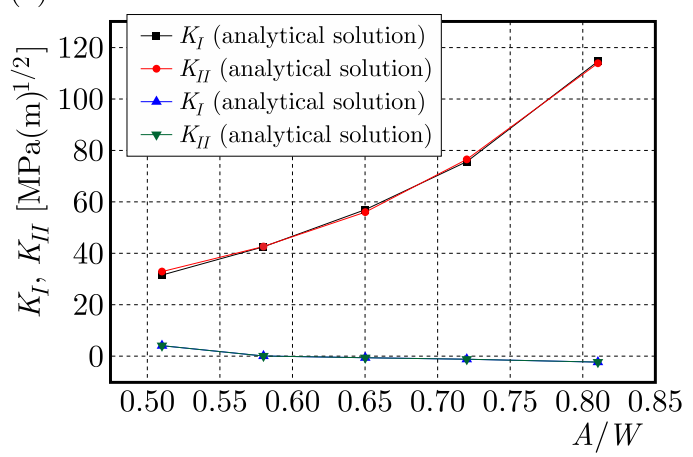

(b)

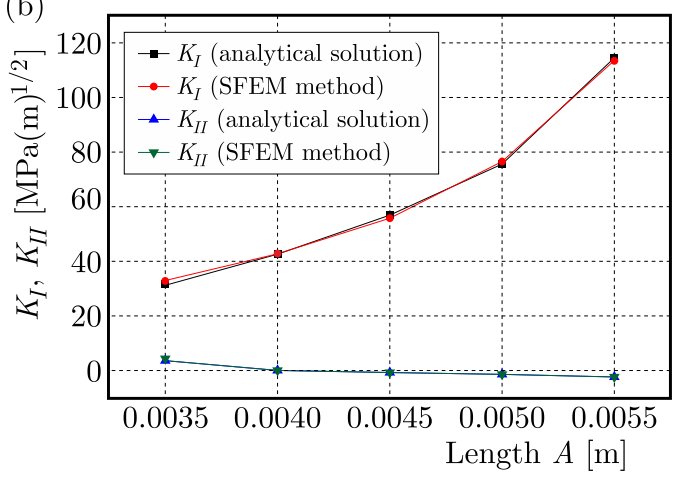

Fig. 7. Comparison of SIF between SFEM and the analytical method: (a) function of the ratio $A / W$, (b) function of the crack length $A$

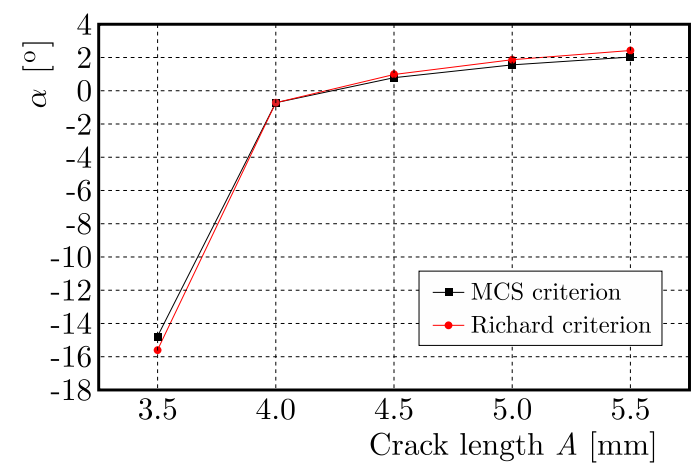

Fig. 8. Evolution of the inclination angle $\alpha$ during crack propagation, comparison between the MCSC and Richard criterion

causes an increase in $K_{I}$ and a decrease in $K_{I I}$. These results were obtained by Boulenouar et al. (2014), Ariffin (2008). The results obtained by the SFEM method and the analytical method show a good correlation between them and allow one to conclude that the numerical model is used correctly.

The values of the stress intensity factors $K_{I}$ and $K_{I I}$ and the orientation angle $\alpha$ are well estimated. Figure 9 illustrates the variation of the estimated angle for each contour of the crack length. There is a very good correspondence between the results obtained by the two methods. In this example, the angle $\alpha$ varies between $-14.79^{\circ}$ and $2.049^{\circ}$. 


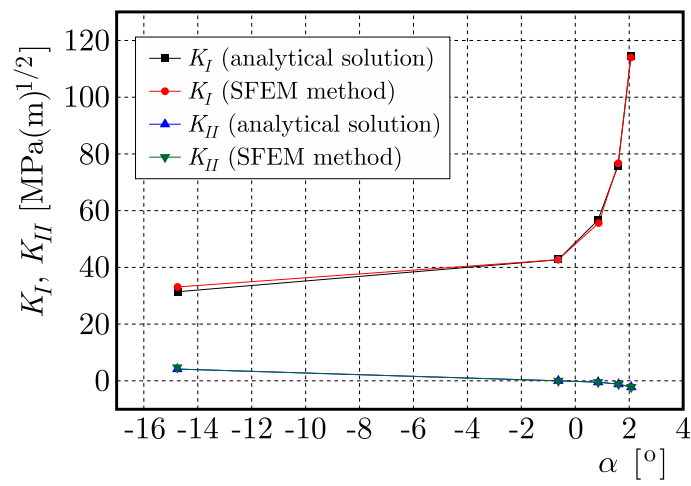

Fig. 9. Tilt angle $\alpha$ according to SIF $\left(K_{I}, K_{I I}\right)$ for the two methods: SFEM and analytical

\section{Conclusion}

The proposed method for the modeling of crack propagation, the stretching finite element method, allows one to get similar results to those obtained through analytical solution. This has been checked for the case in which the analytical solution is well established. The stress intensity factor is rated by the finite element method and its results are in good agreement with those from the exact solution.

Compared (in the case where $A / W=0.5$ to 0.8 ) with the analytical method, the results of the present approach provide favorably comparable values and prove its effectiveness in the modeling of problems with crack propagation. The two MCS criteria and the Richard criterion used to determine the angle of crack orientation show a good correlation between them.

On the other hand the estimation of the error of the stress intensity factors for modes I and II is less than 4.591 per cent. It is assessed as an acceptable threshold. The SFEM method as another finite element method is a function of mesh density. It gives more precision if we increase the number of contours around a singularity point. We have used an example with 5 contours in this study, but our parametric mesh allows us to increase the number of contours, which can improve the results even more. The advantage of this method is keeping the same mesh with the same number of nodes and elements while analyzing crack propagation. It is the only finite element method without remeshing during crack propagation.

\section{References}

1. Aliabadi M.H., 1997, Boundary element formulations in fracture mechanics, Applied Mechanics Reviews, 50, 2, 83-96

2. Alshoaibi M.A., 2015, Finite element modelling of mixed mode crack propagation, International Journal of Soft Computing and Engineering (TM), 5, 5, 61-66

3. Amestoy M., Bui H.D., Dang Van K., 1980, [In:] International Advances in Fracture Research, François D. et al. (Eds.), Oxford, 107-113

4. ARIFfin A.K., 2008, Fatigue life and crack path prediction in $2 \mathrm{G}$ structural components using an adaptive finite element strategy, International Journal of Mechanical and Materials Engineering (IJMME), 3, 1, 97-104

5. Askes H., Sluys L.J., Jong B.B.C., 2001, Remeshing techniques for $r$-adaptive and combined $h / r$-adaptive analysis with application to $2 \mathrm{D} / 3 \mathrm{D}$ crack propagation, International Journal Structural Engineering and Mechanics, 12, 5, 475-490

6. Azocar D., Elgueta M., Rivara M.C., 2010, Automatic LEFM crack propagation method based on local Lepp-Delaunay mesh refinement, Advances in Engineering Software, 41, 111-119 
7. Babuska I., BanerJee U., 2012, Stable generalized finite element method (SGFEM), Computer Methods in Applied Mechanics and Engineering, 201-204, 91-111

8. Babuska I., Belenk J., 1997, The partition of unity method, International Journal Numerical Methods Engineering, 40, 727-758

9. Belytschko T., Gu L., Lu Y.Y., 1994, Fracture and crack growth by element-free Galerkin methods, Modelling and Simulation in Materials Science and Engineering, 2, 3A, 519-534

10. Bouchard P.O., Bay F., Chastel Y., Tovena I., 2000, Crack propagation modelling using an advanced remeshing technique, Computer Methods Applied Mechanics Engineering, 189, 3, 723-742

11. Bouchard P.O., Bay F., Chastel Y., 2003, Numerical modelling of crack propagation automatic remeshing and comparison of different criteria, Computer Methods Applied Mechanics Engineering, 192, 35/36, 3887-3908

12. Boulenouar A., Benseddiq N., Mazari M., Benamara N., 2014, FE model for linear-elastic mixed mode loading: estimation of SIFs and crack propagation, Journal of Theoretical and Applied Mechanics, 52, 2, 373-383

13. Chan S.K., Tuba I.S., Wilson W.K., 1970, On the finite element method in linear fracture mechanics, Engineering Fracture Mechanics, 10, 1, 1-17

14. Chо J.R., 2015, Computation of 2-D mixed-mode stress intensity factors by Petrov-Galerkin natural element method, Structural Engineering and Mechanics, 56, 4, 589-603

15. Duflot M., NGuyen-Dang H., 2004, Fatigue crack growth analysis by an enriched meshless method, Journal of Computational and Applied Mathematics, 168, 1/2, 155-164

16. Erdogan F., Sih G.C., 1963, On the crack extension in plates under plane loading and shear, Journal of Basic Engineering, 85, 4, 519-527

17. Ewalds H., Wanhill R., 1989, Fracture Mechanics, New York, Edward Arnold

18. Khoei A.R., Azadia H., Moslemia H., 2008, Modeling of crack propagation via an automatic adaptive mesh refinement based on modified superconvergent patch recovery technique, Engineering Fracture Mechanics, 75, 2921-2945

19. Meyer A., Rabold F., Scherzer M., 2006, Efficient finite element simulation of crack propagation using adaptive iterative solvers, Communications in Numerical Methods in Engineering, 22, 2, 93-108

20. Miranda A.C.O., Meggiolaro M.A., Castro J.T.P., Martha L.F., Bittencourt T.N., 2003, Fatigue life and crack predictions in generic 2D structural components, Engineering Fracture Mechanics, 70, 10, 1259-1279

21. Möes N., Dolbow J., Belytschko T., 1999, A finite element method for crack growth without remeshing, International Journal Numerical Methods Engineering, 46, 1, 131-150

22. Murat Y., 2016, The investigation crack problem through numerical analysis, Structural Engineering and Mechanics, 57, 6, 1143-1156

23. Nuismer R.J., 1975, An energy release rate criterion for mixed mode fracture, International Journal of Fracture, 11, 2, 245-250

24. PAris P., ERdogan F., 1963, A critical analysis of crack propagation laws, Journal of Basic Engineering, Transactions ASME, 85, 4, 528-534

25. Phongthanapanich S., Dechaumphai P., 2004, Adaptive Delaunay triangulation with objectoriented programming for crack propagation analysis, Finite Elements in Analysis and Design, 40, $13 / 14,1753-1771$

26. Portela A., Aliabadi M., Rooke D.P., 1991, The dual boundary element method effective implementation for crack problem, International Journal for Numerical Methods in Engineering, 33, 6, 1269-1287 
27. Réthoré J., Gravouil A., Combescure A., 2005, An energy-conserving scheme for dynamic crack growth using the extended finite element method, International Journal for Numerical Methods in Engineering, 63, 5, 631-659

28. Sih G.C., Erdogan F., 1962, On crack extension in plants (plates) under plane loading and transverse shear, ASME Meeting WA-163, New York, NY, United States

29. SiH G.C., 1974, Strain-energy-density factor applied to mixed mode crack problems, International Journal of Fracture, 10, 3, 305-321

30. Singh I.V., Mishra B.K., Bhattacharya S., Patil R.U., 2012, The numerical simulation of fatigue crack growth using extended finite element method, International Journal of Fatigue, 36, 1, 109-119

31. TAdA H.P., IRwin G.R., 1985, The Stress Analysis of Cracks Handbook, Paris Productions Incorporated

32. Tada H.P., Paris P.C., Irwin G.R., 2000, The Stress Analysis of Cracks Handbook, American Society of Mechanical Engineering

33. Yan A.M., NguYen-Dang H., 1995, Multiple-cracked fatigue crack growth by BEM, Computational Mechanics, 16, 5, 273-80

34. YAN X., 2006, A boundary element modeling of fatigue crack growth in a plane elastic plate, Mechanics Research Communications, 33, 470-481

35. Zaleha M., Ariffin A.K., Muchtar A., 2007, Prediction of crack propagation direction for holes under quasi-static loading, Computational and Experimental Mechanics, 141-151

Manuscript received December 28, 2016; accepted for print March 16, 2017 